\title{
Time may Heal All Wounds, but While It Does, Melanoma Marches on
}

\author{
David W. Ollila, $\mathrm{MD}^{\mathbf{1}}$, and Michael O. Meyers, $\mathrm{MD}^{\mathbf{1}}$ \\ Department of Surgery, Division of Surgical Oncology, University of North Carolina, Chapel Hill, NC
}

There have long been challenges in managing patients with stage III metastatic melanoma. Surgical resection as the initial therapy has been and remains the standard of care and the primary treatment for these patients; however, many of these patients will recur and some will have very rapid recurrence. ${ }^{1,2}$ In the absence of effective systemic therapy, this has been the reality for the surgeon caring for these patients, despite knowing that some stage III metastatic melanoma patients are unlikely to benefit from aggressive surgical management. The study by Bloemendal et al. ${ }^{3}$ highlights this problem and seeks to characterize a group of patients at greatest risk for early recurrence. The authors present a group of 120 patients with stage IIIb (58\%) and IIIc (43\%) melanoma completely resected surgically and then screened within 10 weeks of surgery for inclusion in an adjuvant therapy trial. Of these patients, $18 \%$ were found to have new disease not identified preoperatively, the majority of whom were asymptomatic. While most patients (83\%) screened for their adjuvant therapy trial had macroscopic nodal disease, an even greater proportion $(91 \%)$ with an early recurrence did, suggesting this clinicopathologic feature in particular may be a primary risk factor. Other common characteristics did not appear to be different between the groups. As the authors point out, these findings underscore the need to closely evaluate patients eligible for adjuvant trials to assure they do not have unsuspected disease prior to initiating therapy. In addition, these results highlight the significant risk of recurrence in this population, the

\footnotetext{
(C) Society of Surgical Oncology 2019
}

First Received: 21 February 2019;

Published Online: 29 August 2019

D. W. Ollila, MD

e-mail: david_ollila@med.unc.edu challenge in managing them surgically, and the critical importance of multimodality therapy in improving outcomes.

Adjuvant therapy for stage III metastatic melanoma has quickly moved away from interferon $\alpha-2 b .{ }^{4}$ With prospective randomized trial data demonstrating improved overall survival and relapse-free survival, the US FDA has approved ipilimumab, ${ }^{5}$ combined dabrafenib/trametinib, ${ }^{6}$ pembrolizumab, ${ }^{7}$ and nivolumab ${ }^{8}$ for patients with surgically resected stage III metastatic melanoma. With the efficacy of new-generation adjuvant therapy, it is not surprising that important data examining neoadjuvant strategies for high-risk, stage III metastatic melanoma patients are being investigated. This includes unresectable stage III metastatic melanoma patients and/or patients with bulky adenopathy, but more recently has begun to examine patients with clearly resectable disease. A natural extension of improvements in adjuvant therapy in many diseases, neoadjuvant therapy has the potential to improve $R_{0}$ resection rates, and, importantly, may serve to select those patients who might benefit most from aggressive surgical resection.

Specifically in melanoma, an immunological tumor, another important consideration for neoadjuvant therapy is the potential for a greater $\mathrm{T}$ cell response when treatment with T-cell checkpoint inhibitors are used while bulkier disease is still present. ${ }^{9}$ These early data have included evaluation of both BRAF-mutated melanoma and, in other studies, immune-checkpoint treatment regardless of BRAF status. ${ }^{10-12}$ To date, these have all been phase Ib or II trials and the number of patients treated have been small, but, importantly, all of these studies have included patients with resectable regional disease and not just patients with marginally or unresectable melanoma. The first of these trials randomized patients with BRAF-mutated melanoma to neoadjuvant dabrafenib and trametinib for 8 weeks prior to 
surgery and continue the same therapy postoperatively for up to 52 weeks of treatment. ${ }^{10}$ Control patients were treated with 'standard of care', which included surgical resection as the initial treatment for all patients. Toxicity was acceptable and all but one patient in the treatment arm proceeded to surgery. Median event-free survival was markedly better in the treatment arm (19.7 months vs. 2.9 months), although confounded by uncommon use of systemic therapy in the control arm, and the trial was stopped early by the Data Safety Monitoring Board. This was a small $(n=21)$, nonrandomized clinical trial, but, nonetheless, is an important proof of concept and clear evidence that neoadjuvant therapy does not compromise care.

More recently, two small studies have been reported examining neoadjuvant therapy with immune-checkpoint blockade. ${ }^{11,12}$ One of these studies randomized patients $(n=20)$ to neoadjuvant versus adjuvant nivolumab + ipilimumab. ${ }^{11}$ Both groups received four courses of nivolumab + ipilimumab, with the neoadjuvant group receiving two courses before surgery. Interpreting survival outcomes in this small trial is challenging, but the pathologic response to neoadjuvant therapy was significant in the majority (78\%), with three pathologic complete responses and three 'near complete' responses. The other immune checkpoint inhibitor trial $(n=23)$ compared neoadjuvant nivolumab (up to four treatments) with nivolumab + ipilimumab (up to three treatments), followed by nivolumab after surgical resection. Similarly, the combination of nivolumab + ipilimumab yielded significant pathologic response rates $(45 \%$ pathologic complete response/73\% radiographic response) compared with nivolumab alone (25\% pathologic and radiographic response). This trial was stopped early because of disease progression in two patients in the single-agent nivolumab arm that precluded surgery, as well as concerns about significant toxicity. Collectively, these studies at least lay the foundation for neoadjuvant therapy, although toxicity remains a concern and identifying the optimal regimen to balance efficacy and toxicity remains elusive. To that end, a recently reported phase II trial of resectable stage III melanoma compared three neoadjuvant regimens of ipilimumab and nivolumab. ${ }^{13}$ Toxicity was improved in the two combined therapy arms compared with the sequential therapy arm (ipilimumab first followed by nivolumab), as was pathologic complete response (47-57\% vs. $25 \%$ ). As a whole, these studies appear to suggest that it is safe to begin treatment for regionally advanced but resectable melanoma with systemic therapy as opposed to surgery first. While optimal treatment strategies remain to be defined and the efficacy of neoadjuvant therapy compared with adjuvant therapy is as yet unclear, this shows promise in a patient population that has substantial recurrence risk.
Bloemendal and colleagues are to be commended for reporting, in a rigorous fashion, the number of patients with surgically resected, high-risk, stage III metastatic melanoma who were screened prior to entering an adjuvant clinical trial. ${ }^{3}$ The systematic precision of restaging before embarking on adjuvant therapy is proper clinical trial conduct; however, the real message from this work may be the high number of patients $(18 \%)$ who were found to have disease within 7.5 weeks of their definitive surgical resection of stage III metastatic melanoma. This alarming number of patients who, on restaging, were found to have recurrent stage III or even stage IV disease highlights the need for a paradigm shift to neoadjuvant therapy for these high-risk patients. Instead of continuing with a surgery-first approach, we believe the work of Bloemendal et al. ${ }^{3}$ highlights the need to shift to a drug-first approach. We would encourage investigators to continue their thoughtful use of checkpoint hihibitors, BRAF and MEK inhibitors, and/or novel combinations in the neoadjuvant setting as opposed to the adjuvant setting for patients with high-risk stage III metastatic melanoma.

\section{REFERENCES}

1. Murali R, Moncrieff MD, Hong J, et al. The prognostic value of tumor mitotic rate and other clinicopathologic factors in patients with locoregional recurrences of melanoma. Ann Surg Oncol. 2010;17(11):2992-9.

2. Baker JJ, Ollila DW, Deal AM, Frank J, Amos KD, Meyers MO. Early recurrence in sentinel lymph node positive stage III melanoma patients. Am Surg. 2012;78(7):808-13.

3. Bloemendal M, van Willigen WW, Bol KF, et al. Early recurrence in completely resected iiib and iiic melanoma warrants restaging prior to adjuvant therapy. Ann Surg Oncol. Epub 4 Mar 2019. https://doi.org/10.1245/s10434-019-07274-2.

4. Kirkwood JM, Strawderman MH, Ernstoff MS, Smith TJ, Borden $\mathrm{EC}$, Blum RH. Interferon alfa-2b adjuvant therapy of high-risk resected cutaneous melanoma: the Eastern Cooperative Oncology Group Trial EST 1684. J Clin Oncol. 1996;14(1):7-17.

5. Eggermont AM, Chiarion-Sileni V, Grob JJ, et al. Prolonged survival in stage III melanoma with ipilimumab adjuvant therapy. $N$ Engl J Med. 2016;375(19):1845-55.

6. Long GV, Hauschild A, Santinami M, et al. Adjuvant dabrafenib plus trametinib in stage III BRAF-mutated melanoma. $N$ Engl $J$ Med. 2017;377(19):1813-23.

7. Eggermont AMM, Blank CU, Mandala M, et al. Adjuvant pembrolizumab versus placebo in resected stage III melanoma. $N$ Engl J Med. 2018;378(19):1789-801.

8. Weber J, Mandala M, Del Vecchio M, et al. Adjuvant nivolumab versus ipilimumab in resected stage III or IV melanoma. $N$ Engl J Med. 2017;377(19):1824-35.

9. Liu J, Blake SJ, Yong MC, et al. Improved efficacy of neoadjuvant compared to adjuvant immunotherapy to eradicate metastatic disease. Cancer Discov. 2016;6(12):1382-99.

10. Amaria RN, Prieto PA, Tetzlaff MT, et al. Neoadjuvant plus adjuvant dabrafenib and trametinib versus standard of care in patients with high-risk, surgically resectable melanoma: a singlecentre, open-label, randomised, phase 2 trial. Lancet Oncol. 2018;19(2):181-93. 
11. Blank CU, Rozeman EA, Fanchi LF, et al. Neoadjuvant versus adjuvant ipilimumab plus nivolumab in macroscopic stage III melanoma. Nat Med. 2018;24(11):1655-61.

12. Amaria RN, Reddy SM, Tawbi HA, et al. Neoadjuvant immune checkpoint blockade in high-risk resectable melanoma. Nat Med. 2018;24(11):1649-54.

13. Blank CU, Rozeman EA, Menzies AM, et al. OpACIN-neo: a multicenter phase II study to identify the optimal neo-adjuvant combination scheme of Ipilimumab (IPI) and nivolumab (NIVO). Ann Oncol. 2018;29(Issue Suppl 8).

Publisher's Note Springer Nature remains neutral with regard to jurisdictional claims in published maps and institutional affiliations. 